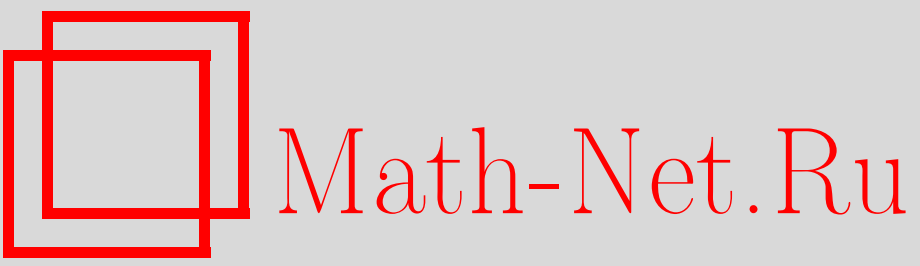

С. Ю. Доброхотов, Интегрирование в квадратурах $2 n$-мерных линейных гамильтоновых систем с $n$ известными косоортогональными решениями, $У M H$, 1998, том 53, выпуск 2, 143-144

DOI: https://doi.org/10.4213/rm34

Использование Общероссийского математического портала Math-Net.Ru подразумевает, что вы прочитали и согласны с пользовательским соглашением

http: //www . mathnet.ru/rus/agreement

Параметры загрузки:

IP : 3.82 .47 .9

26 апреля 2023 г., 17:59:32 


\title{
ИНТЕГРИРОВАНИЕ В КВАДРАТУРАХ $2 n$-МЕРНЫХ ЛИНЕЙНЫХ ГАМИЛЬТОНОВЫХ СИСТЕМ С $n$ ИЗВЕСТНЫМИ КОСООРТОГОНАЛЬНЫМИ РЕШЕНИЯМИ
}

\author{
С. Ю. Доврохотов
}

Предположим,что у гамильтоновой системы $\dot{x}=J H_{x}(x), J=\left(\begin{array}{cc}0 & -E_{n} \\ E_{n} & 0\end{array}\right)$ в $2 n$-мерном фазовом пространстве $\mathbb{R}_{x}^{2 n}$ с гамилтонианом $H(x) \in \mathbb{C}^{2}\left(\mathbb{R}_{x}^{2 n}\right)$ имеется $n$-мерное инвариантное лагранжево многообразие (компактное или некомпактное) $\Lambda=\left(x \in \mathbb{R}_{x}^{2 n}: x=X(\varphi)\right)$ с накрьвающей, диффеоморфной $\mathbb{R}^{n}$, и с координатами (на накрьвающей) $\varphi=\left(\varphi_{1}, \ldots, \varphi_{n}\right) \in \mathbb{R}^{n}$, такими что ее траектории на $\Lambda$ имеют вид $x=X(\varphi+\omega t)$, где $\omega=\left(\omega_{1}, \ldots, \omega_{n}\right) \in \mathbb{R}^{n}$-вектор "частот". Мы обозначаем через $E_{n} n \times n$ единичную матрицу, и через 0 матрицу нужного размера с нулевыми элементами. Условие лагранжевости означает, что в любой точке $\varphi$ все касательные векторы $\partial X / \partial \varphi_{j}$ косоортогональны друг другу: $\left(X_{\varphi_{j}}, J X_{\varphi_{k}}\right)=0$ для всех $j, k=1, \ldots, n$ (см., например, [1], [3], [4]). Эти векторы линейно-независимы в силу условия $\operatorname{dim} \Lambda=n$. Гамильтонова система и траектории $x=X(\varphi+\omega t)$ порождают систему в вариациях $\dot{z}=J A(t) z$, где $A=\| \partial^{2} H / \partial x_{i} \partial x_{j}(X(\varphi+\omega t) \|$.

Хорошо известно (и очевидно), что вектор-функции $Y_{j}(t)=X_{\varphi_{j}}(\varphi+\omega t)$ являются решениями этой системы. Из общей теории линейных систем следует, что знание $n$ линейно-независимых решений $n$-мерной линейной системы позволяет понизить ее порядок на $n$. Оказьвается, что для системы в вариациях знание решений $Y_{j}$ позволяет явно (в квадратурах) построить все остальные ее решения. Хотя этот факт представляется весьма естественным, его доказательство элементарно, и он в неявной форме, вероятно, использовался в работах, связанных с теорией возмущений гамиль тоновых систем и геометрическим квантованием, тем не менее ни мне, ни многим специалистам в области гамильтоновых систем соответствующие формулы не были известны.

Введем $2 n \times n$ матрицу $Y(t)=\left(Y_{1}, \ldots, Y_{n}\right)$ и $n \times n$ невырожденную матрицу ${ }^{t} Y(t) Y(t)$, где индекс $t$ слева вверху означает транспонирование. В силу косоортогональности векторов $Y_{1}, \ldots, Y_{n}$ справедливо равенство ${ }^{t} Y J Y=0$. Обозначим через $M(t) n \times n$ матрицу $M(t)=$ $\left({ }^{t} Y(t) Y(t)\right)^{-1}$ и через $[A, J]=A J-J A$. Далее определим $n \times n$ матрицу

$$
R(t)=\int_{0}^{t} M(\xi)^{t} Y(\xi) J[A(\xi), J] Y(\xi) M(\xi) d \xi
$$

и $(2 n \times 2 n)$-матрицу $\Phi(t)=(Y(t), Y(t) R(t)+J Y(t) M(t))$.

Tеорема 1. Матрица $\Phi(t)$ есть фундаментальная матрица системы $\dot{z}=J A(t) z$.

ДокАЗАТЕЛЬСтво. Очевидно каждый из векторов $Y_{i}(t), i=1, \ldots, n$, ортогонален каждому из векторов $J Y_{j}(t), j=1, \ldots, n$. Поэтому совокупность этих векторов образует базис в евклидовом пространстве $\mathbb{R}_{x}^{2 n}$. Так как $Y_{j}$ являются решениями системы в вариациях, то фундаментальная матрица может быть представлена в виде, указанном в теореме, с некоторыми матрицами $M(t)$ и $R(t)$. Выберем матрицу $M(t)$ таким образом, чтобы при $t=0$ б была симплектичной, т.е. чтобы ${ }^{t} \Phi J \Phi=J$. В силу гамильтоновости линейной системы в вариациях это свойство сохраняется для всех $t$ (см., например, [1], [2].) Отсюда немедленно следует равенство $M(t)=\left({ }^{t} Y(t) Y(t)\right)^{-1}$. Теперь нам осталось подставить в систему в вариациях матричнозначную функцию $Y(t) R(t)+J Y(t) M(t)$ и потребовать, чтобы она была ее решением. Учитывая,что $Y(t)$ есть решение этой системы, умножая затем полученное равенство на $M^{t} Y$ слева, и интегрируя резулштат приходим к $(1)$.

ЗАмечАниЕ 1 . Из доказательства ясно, что теорема справедлива для любой линейной гамилштоновой системы $\dot{z}=J A(t) z \mathrm{c} A(t)={ }^{t} A(t)$, у которой известно $(2 n \times n)$-матричное решение, вектор-столбцы которого линейно независимы и косоортогональны. В частности теорема справедлива для системы второго порядка $(n=1)$ с коэффициентами $a_{12}=0, a_{11}=1$,

Работа выполнена при поддержке Российского фонда фундаментальных исследований (грант № 96-01-00720). 
$a_{22}=a(t)$. Если обозначить $v=y_{2}, \dot{v}=y_{1}$, то для $v$ имеем уравнение $\ddot{v}+a(t) v=0$. Пусть $v_{1}(t)$ - некоторое его решение, тогда второе линейно независимое решение имеет вид

$$
v_{2}=v_{1} \int_{0}^{t}(a(t)-1)\left(v_{1}^{2}-\dot{v}_{1}^{2}\right) /\left(v_{1}^{2}+\dot{v}_{1}^{2}\right)^{2} d t+\dot{v}_{1} /\left(v_{1}^{2}+\dot{v}_{1}^{2}\right)
$$

По сравнению с хорошо известной формулой для второго решения $v_{2}=v_{1} \int d t / v_{1}^{2}$, эта формула имеет более громоздкий вид, но в отличие от последней она годится (без дополнительных исследований) в том случае, когда $v_{1}$ обращается в ноль в некоторых точках.

ЗАмечАниЕ 2 . Пусть $\Lambda$ - тор и система $\dot{x}=J H_{x}$ вполне интегрируема в некоторой окрестности $\Lambda$. Тогда в ней можно ввести переменные действия $I=\left(I_{1}, \ldots, I_{n}\right)$, сопряженные с $\varphi$ (см. [1]); считаем, что равенство $I=I^{0}$ задает $\Lambda$. Матрица $W(\varphi, t)=X_{I}(\varphi+\omega t)+t X_{\varphi}(\varphi+$ $\omega t) \omega_{I}$, где $\omega_{I}=\left\|\partial \omega_{i} / \partial I_{j}\right\|, i, j=1 \ldots, n$, является матричным решением системы вариациях. С учетом симплектичности матрицы $\left(X_{\varphi}, X_{I}\right)$ имеем $X_{I}(\varphi)=J X_{\varphi} \widetilde{M}(\varphi)+X_{\varphi}(\varphi) Q(\varphi)$, где $\widetilde{M}(\varphi)=\left({ }^{t} X_{\varphi}(\varphi) X_{\varphi}(\varphi)\right)^{-1}$ и $Q(\varphi)$ - гладкая $(n \times n)$-матричнозначная функция. Очевидно, $W(\varphi, t)-X_{\varphi}(\varphi+\omega t) Q(\varphi)$ есть $(2 n \times n)$-матричное решение этой же системы, совпадающее со вторым блоком фундаментального решения $\Phi$. Отсюда для $I=I^{0}$ немедленно следует ("гомологическое") уравнение для определения $Q(\varphi)$ (и тем самым для $\left.\left.X_{I}(\varphi)\right|_{I=I^{0}}\right)$ и формула для $\left.\omega_{I}\right|_{I=I^{0}}$ :

$$
\begin{gathered}
Q(\varphi+\omega t)=Q(\varphi)+\left.\int_{0}^{t} \widetilde{M}^{t} X_{\varphi} J\left[H_{x x}, J\right] X_{\varphi} \widetilde{M}\right|_{\varphi^{\prime}=\varphi+\omega \xi} d \xi-t \omega_{I}, \\
\left.\left.\omega_{I}\right|_{I=I^{0}}=\lim _{t \rightarrow \infty} \frac{1}{t} \int_{0}^{t} \widetilde{M}^{t} X_{\varphi} J\left[H_{x x}, J\right]\right)\left.X_{\varphi} \widetilde{M}\right|_{\varphi^{\prime}=\varphi+\omega \xi} d \xi
\end{gathered}
$$

В "вырожденных" ситуациях гамилтоновы системы допускают лишь $k$-мерные изотропные многообразия $(k<n)$, движение на которых по-прежнему имеет вид $x=X(\varphi+\omega t)$, но $\varphi$ и $\omega$ в этих формулах $k$-мерные векторы и соответствующая матрица $Y=X_{\varphi}$ состоит из $k$ линейно независимых попарно косоортогональных векторов. Часто, однако, удается построить еще $n-k$ комплексных линейно независимых решений и $n-k$ комплексно сопряженных к ним (см. [3], [4]). Таким образом, оказьвается известно $2 n-k$ линейно независимых решений, образующих $2 n \times(n-k)$ матрицы $Z(t)$ и $\bar{Z}(t)$ и $2 n \times k$ матрицу $Y(t)$. Они нормированы условиями ${ }^{t} \bar{Z} J Z=2 i E_{n-k},{ }^{t} Z J Y=O,{ }^{t} Z J Z=O$. В этом случае “недостающий" блок в фундаментальной матрице $\Phi(t)=(\bar{Z}, Z, Y, U)-(2 n \times k)$-матрицу $U(t)$ тоже удается выразить в квадратурах, хотя и с помощью более сложной (по виду) формулы. Введем $(k \times k)$-матрицу $M(t)=\left({ }^{t} Y(t) Y(t)\right)^{-1},((n-k) \times k)$-матрицу $G(t)=\frac{i}{2}^{t} Z Y M$ и $(k \times k)$-матрицу

$$
R=\int_{0}^{t} M^{t} Y\left(J-\frac{i}{2}\left(\bar{Z}^{t} Z-Z^{t} \bar{Z}\right)\left(E_{2 n}-Y M^{t} Y\right)\right)[A, J] Y M d \xi
$$

Теорема 2. Положим $U=\bar{Z} G+Z \bar{G}+Y R+J Y M$. Тогда при сформулированных выше предположения $\Phi(t)=(\bar{Z}, Z, Y, U)$ есть фундаментальная матрица системь $\dot{z}=J A(t) z$, при әтом ${ }^{t} \bar{Z} J U={ }^{t} Z J U=O,{ }^{t} Y J U=E_{k}$.

\section{СПИСОК ЛИТЕРАТУРЫ}

[1] Арнольд В.И., Козлов В.В., Нейштадт А.И. Математические аспекты классической и небесной механики // Итоги науки и техники. Совр. пробл. матем. Фунд. напр. Т. 3. М.: ВИНИТИ, 1985. С. 5-300. [2] Якубович В. А., Старжинский В. М. Линейные дифференциальные уравнения с периодическими коэффициентами и их приложения. М.: Наука, 1972. [3] Маслов В. П. Комплексньй метод ВКБ в нелинейных уравнениях. М.: Наука, 1977. [4] Нехорошев Н. Н. // Труды ММО. 1972. Т. 26. С. 181-198. 